Pacific Journal of Mathematics

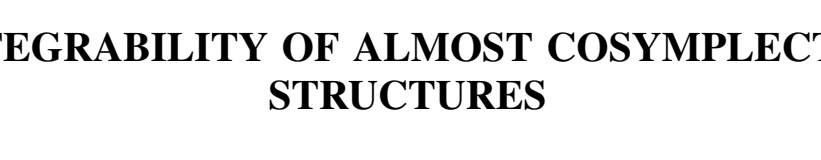




\title{
INTEGRABILITY OF ALMOST COSYMPLECTIC STRUCTURES
}

\author{
Samuel I. Goldberg and Kentaro Yano
}

\begin{abstract}
Integrability conditions for almost cosymplectic structures on almost contact manifolds are obtained. Examples of these structures are given by taking the direct product of an almost Kaehler manifold with a line $R$ or a circle $S^{1}$. If the curvature transformation of the metric associated with an almost cosymplectic space $M$ commutes with the fundamental singular collineation $\phi$ of $M$, then the related almost contact structure on $M$ gives rise to a complex structure on $M \times R$. The manifold $M$ is then a cosymplectic space, examples being given by taking the direct product of a Kaehler manifold with $R$ or $S^{1}$. In particular, an almost cosymplectic manifold is cosymplectic if and only if it is locally flat.
\end{abstract}

In a recent paper [3] one of the authors examined the integrability of almost Kaehler manifolds $M(J, g)$ showing, in particular that if the curvature transformation of the almost Kaehler metric $g$ commutes with the almost complex structure tensor $J$, then $J$ is integrable, that is, the structure $(J, g)$ on $M$ is Kaehlerian. This is also a special case of a theorem due to A. Gray [4] whose methods apparently do not extend to include the results of this paper which, therefore, complement those given by him. It was also proved that an almost Kaehler space of constant curvature is a Kaehler space if and only if it is locally fiat. Our main purpose here is to extend these results to almost cosymplectic manifolds.

THEOREM 1. If the curvature transformation of the metric $g$ of the almost cosymplectic manifold $M(\phi, \eta, g)$ commutes with $\phi$, then $M$ is normal, that is, it is a cosymplectic manifold.

A cosymplectic manifold of constant curvature is locally flat in the given metric [1]. For almost cosymplectic spaces we have

CoROLlaRY 1.1. An almost cosymplectic manifold of constant curvature is cosymplectic if and only if it is locally flat.

By imposing a condition on the scalar curvature of an almost cosymplectic space, the same conclusion prevails. Examining the Nijenhuis torsion of the collineation $\phi$, we find that a 3-dimensional almost cosymplectic manifold is cosymplectic if its fundamental vector field is a Killing field. 
In $\S 5$, integrability conditions for almost Sasakian manifolds are also given.

The manifolds considered in the sequel are $C^{\infty}$ and connected.

2. Almost contact manifolds. The reader is referred to [1] for more details on this section, only the bare essentials being presented here. A $(2 n+1)$-dimensional manifold $M$ having the property that the structural group of its tangent bundle is reducible to $U(n) \times 1$ is called an almost contact manifold. Several tensor fields are thereby distinguished, namely, a linear transformation field $\phi$ acting in each tangent space $M_{m}$ of $M, m \in M$, called the fundamental singular collineation, a vector field $\xi$ on $M$ called the fundamental vector field, and a contact form $\eta$ such that

$$
\begin{aligned}
\eta(\xi) & =1, & \phi \xi & =0, \\
\eta \circ \phi & =0, & \phi^{2} & =-I+\xi \otimes \eta .
\end{aligned}
$$

An almost contact manifold $M$ admits a Riemannian metric $g$ such that

$$
\begin{aligned}
g(\phi X, Y) & =-g(X, \phi Y), \\
g(X, \xi) & =\eta(X),
\end{aligned}
$$

and in this case we denote the manifold by $M(\phi, \eta, g)$. A 2-form $\Phi$ called the fundamental form of $M(\phi, \eta, g)$ is defined by

$$
\Phi(X, Y)=g(\phi X, Y) \text {. }
$$

From (2.1) and (2.2) it is easily seen that $|\Phi|^{2} \equiv\langle\Phi, \Phi\rangle=2 n$ where $\langle$, $\rangle$ denotes the local scalar product induced by $g$.

If $M(\phi, \eta, g)$ is a contact structure, its fundamental form is exact. In fact,

$$
\Phi=d \eta
$$

If the fundamental vector field of a contact metric structure is a Killing field with respect to its contact metric, the manifold is said to be almost Sasakian. An almost contact metric manifold $M(\phi, \eta, g)$ is called almost cosymplectic if both its fundamental form and contact form are closed, that is, if

$$
d \Phi=0 \text { and } d \eta=0 .
$$

An almost contact manifold $M(\phi, \eta, g)$ is said to be normal if the tensor field $[\phi, \phi]+d \eta \otimes \xi$ vanishes where

$$
[\phi, \phi](X, Y)=[\phi X, \phi Y]-\phi[\phi X, Y]-\phi[X, \phi Y]+\phi^{2}[X, Y] \text {. }
$$


A normal contact metric manifold is called a Sasakian manifold. It is easily shown that the fundamental vector field of a Sasakian manifold is a Killing field. If $M$ is almost cosymplectic, $d \eta=0$, so the normality condition is given by the vanishing of the torsion tensor $[\phi, \phi]$, and in this case, $M$ is said to be cosymplectic. If $M(\phi, \eta, g)$ is Sasakian, then $\nabla \xi=\phi$ and $\nabla_{X} \phi=-g(X, \cdot) \xi+\eta \otimes X$, where $\nabla$ denotes covariant differentiation with respect to the Riemannian connection. If $M(\phi, \eta, g)$ is cosymplectic, both $\nabla \eta$ and $\nabla \phi$ vanish [1].

3. The curvature transformation of an almost contact metric manifold. Let $M(\phi, \eta, g)$ be an almost contact metric manifold. An orthonormal basis $\left\{X_{0}, X_{1}, \cdots, X_{2 n}\right\}$ on $M_{m}$ with $X_{0}=\xi$ and $X_{n+i}=$ $\phi X_{i}, i=1, \cdots, n$ is called a $\phi$-basis of $M_{m}$. In the sequel, we set $i^{*}=n+i$.

LEMMA 1 (Moskal [5]). Let $M(\phi, \eta, g)$ be an almost contact metric manifold. Then, for every $m \in M$, there is a $\phi$-basis of $M_{m}$.

The relationship between the curvature transformation $R(X, Y), X$, $Y \in M_{m}$ and the metric is given by

$$
R(X, Y)=\left[\nabla_{X}, \nabla_{Y}\right]-\nabla_{[X, Y]} \cdot
$$

We denote by $K(X, Y)$ the sectional curvature of the plane determined by the vectors $X$ and $Y$.

Lemma 2. Let $M(\phi, \eta, g)$ be a cosymplectic manifold. Then, for any $X, Y \in M_{m}$,

(a) $R(\phi X, \phi Y)=R(X, Y)$,

(b) $K(\phi X, \phi Y)=K(X, Y)$,

(c) $K(X, \xi)=0$,

and when $X, Y, \phi X, \phi Y$ are orthonormal vectors

(d) $g(R(X, \phi X) Y, \phi Y)=-K(X, Y)-K(X, \phi Y)$.

Proof. Applying the Ricci interchange formula to the tensor field $\phi$ and employing (2.1), we obtain (a); to prove (b) and (d) apply the usual symmetry properties of the curvature tensor. The relationship (c) is a consequence of (a).

In terms of a basis $\left\{X_{\alpha}\right\}_{\alpha=0,1, \ldots, 2 n}$ of $M_{m}$ we put

$$
\begin{aligned}
R_{\alpha \beta \gamma \delta} & =g\left(R\left(X_{\alpha}, X_{s}\right) X_{\gamma}, X_{\delta}\right), \\
R_{\alpha \beta} & =\operatorname{trace}\left(X_{\gamma} \rightarrow-R\left(X_{\alpha}, X_{\gamma}\right) X_{\beta}\right), \\
\zeta_{\alpha_{1} \cdots \alpha_{p}} & =\zeta\left(X_{\alpha_{1}}, \cdots, X_{\alpha_{p}}\right),
\end{aligned}
$$

and denote the curvature and Ricci tensors by $R$ and $S$, respectively. 
If $\left\{X_{\alpha}\right\}$ is an orthonormal basis, the codifferential ${ }^{1} \delta \omega$ at $m$ of a $p$ form $\omega$ is defined by

$$
(\delta \omega)\left(Y_{1}, \cdots, Y_{p-1}\right)=-\sum_{i}\left(\nabla_{X_{i}} \omega\right)\left(X_{i}, Y_{1}, \cdots, Y_{p-1}\right) .
$$

The operator $\delta$ is the adjoint of $d$, that is $\delta \omega=(-1)^{p} * d * \omega$ where $*$ is the Hodge star operation. A differential form is harmonic if it is a zero of the operators $d$ and $\delta$. We shall denote by $\Delta$ the LaplaceBeltrami operator $d \delta \hat{\delta}+\delta d$.

Lemma 3. The contact form $\eta$ and the fundamental form $\Phi$ of an almost cosymplectic manifold are harmonic forms.

Proof. By ([2], Proposition 2.12) the forms $\eta$ and $\Phi$ are related by

$$
\eta= \pm \frac{1}{\left|\Phi^{n}\right|} * \Phi^{n}
$$

Hence, since $|\Phi|$ is constant and $\Phi$ is closed,

$$
\begin{aligned}
\delta \eta & = \pm \frac{1}{\left|\Phi^{n}\right|} * d \Phi^{n} \\
& =0 .
\end{aligned}
$$

On the other hand, $\iota(\Phi) d \Phi=0$, where $\iota$ is the interior product operation, from which $\iota(\Phi) \nabla \Phi=0$. But

$$
\begin{aligned}
\frac{1}{2} \iota(\Phi) \nabla \Phi & =\iota(\delta \Phi) \Phi-\delta \eta \cdot \eta+\iota(\eta) \nabla \eta \\
& =\iota(\delta \Phi) \Phi+\frac{1}{2} d|\eta|^{2}=\iota(\delta \Phi) \Phi,
\end{aligned}
$$

and consequently $\iota(\delta \Phi) \Phi=0$, since $\delta \eta=0$ and $|\eta|=1$. Applying $\phi$ to $\iota(\delta \Phi) \Phi$ we get $\delta \Phi+\eta \otimes \iota(\xi) \delta \Phi=\delta \Phi$ since $\iota(\xi) \Phi$ vanishes and $\eta$ is closed. Hence, $\Phi$ is coclosed.

The following lemma is required in the proof of Theorem 1 (see [3], Proposition 2).

Lemma 4. A harmonic p-form of constant length on a Riemannian manifold has vanishing covariant derivative if and only if the quadratic form

$$
F(\zeta)=R_{\alpha \beta} \zeta^{\alpha \alpha_{2} \cdots \alpha_{p} \zeta^{\beta}{ }_{\alpha_{2} \cdots \alpha_{p}}}+\frac{p-1}{2} R_{\alpha \beta \gamma \delta} \zeta^{\alpha \beta \alpha_{3} \cdots \alpha_{p} \zeta \zeta^{\gamma \delta}{ }_{\alpha_{3} \cdots \alpha_{p}}}
$$

1 The codifferential of a tensor field of type $(0, p)$ may be similarly defined (see $\S 5)$. 
is non negative.

A 2-form $\zeta$ on an almost contact metric manifold $M(\phi, \eta, g)$ is said to be of bidegree $(1,1)$ if $\zeta(X, \phi Y)+\zeta(\phi X, Y)=0$. The following result is due to Moskal [5].

Lemma 5. A $\phi$-basis $\left\{X_{0}, X_{i}, \phi X_{i}\right\}_{i=1, \ldots, n}$ may be chosen at each point of an almost contact metric manifold $M(\phi, \eta, g)$ such that the only nonvanishing components of a 2 -form $\zeta$ of bidegree $(1,1)$ are of the type $\zeta_{i i^{*}}=\zeta\left(X_{i}, \phi X_{i}\right)$.

For the components of the 2-form $\Phi$, we have

$$
\begin{aligned}
\phi_{i j}= & \Phi\left(X_{i}, X_{j}\right)=-g\left(X_{i}, \phi X_{j}\right)=0, \\
\phi_{i j^{*}}= & \Phi\left(X_{i}, X_{j^{*}}\right)=-g\left(X_{i}, \phi^{2} X_{j}\right)=g\left(X_{i}, X_{j}\right)=\delta_{i j}, \\
\phi_{i^{*} j^{*}}= & \Phi\left(X_{i^{*}}, X_{j^{*}}\right)=-g\left(\phi X_{i}, \phi^{2} X_{j}\right)=-g\left(X_{i}, \phi X_{j}\right) \\
& +\eta\left(X_{i}\right) \eta\left(\phi X_{j}\right)=0, \\
\phi_{0 \alpha}= & \Phi\left(X_{0}, X_{\alpha}\right)=-g\left(X_{0}, \phi X_{\alpha}\right)=0, \quad \alpha=1, \cdots, 2 n .
\end{aligned}
$$

Proposition 2. Let $\zeta$ be a harmonic form of bidegree $(1,1)$ on the almost contact manifold $M$. Then, the quadratic form $F(\zeta)$ on $M$ may be expressed in the canonical form

$$
\begin{aligned}
2 F(\zeta)= & \sum_{i} \sum_{j \neq i, i^{*}}\left(2 K_{i 0}+K_{i j}+K_{i j^{*}}+K_{i^{*} j}+K_{i^{*} j^{*}}\right)\left(\zeta_{i i^{*}}\right)^{2} \\
& +8 \sum_{i<j} R_{i i^{*} j j^{*}} \zeta_{i i^{*}} \zeta_{j j^{*}}
\end{aligned}
$$

where $K_{\alpha \beta}=K\left(X_{\alpha}, X_{\beta}\right)$.

CoROLlary 2.1. If $M$ is cosymplectic and curvature is nonnegative the covariant derivative of a harmonic 2 -form of bidegree $(1,1)$ and constant length vanishes.

This is an immediate consequence of the identity $(d)$ in $\S 3$.

COROLLARY 2.2. The covariant derivative of a harmonic form of bidegree $(1,1)$ on a homogeneous cosymplectic space of nonnegative curvature with respect to the invariant metric vanishes.

4. Proof of Theorem 1. Since

$$
g(R(X, Y) \phi Z, W)=g(\phi R(X, Y) Z, W)=-g(R(X, Y) Z, \phi W),
$$

we have $g(R(\phi Z, W) X, Y)=-g(R(Z, \phi W) X, Y)$ for all $X$ and $Y$. Hence $R(\phi Z, W)=-R(Z, \phi W)$. For sectional curvature we have the 
corresponding relation $K(X, \phi Y)=K(Y, \phi X)$. When $X, Y, \phi X$ and $\phi Y$ form an orthonormal set

$$
\begin{aligned}
g(R(X, \phi X) Y, \phi Y) & =g(R(X, Y) \phi X, \phi Y)+g(R(X, \phi Y) X, \phi Y) \\
& =-g\left(R(X, Y) X, \phi^{2} Y\right)-K(X, \phi Y) \\
& =-K(X, Y)-\eta(Y) g(R(X, Y) X, \xi)-K(X, \phi Y) \\
& =-K(X, Y)-K(X, \phi Y)
\end{aligned}
$$

since $g(R(X, Y) X, \xi)=0$, the latter statement following from the fact that $g(R(X, Y) \phi Z, \xi)=-g(R(X, Y) Z, \phi \xi)=0$. For any tangent vector $X, K(X, \xi)=0$ since $R(\phi X, Y)=-R(X, \phi Y)$.

Let $\zeta$ be a harmonic form of bidegree $(1,1)$ on the almost cosymplectic manifold $M$. Then, the quadratic form $F(\zeta)$ on $M$ may be expressed in the normal form

$$
\begin{aligned}
F(\zeta) & =\sum_{i} \sum_{j \neq i, i^{*}}\left(K_{i j}+K_{i j^{*}}\right)\left(\zeta_{i i^{*}}\right)^{2}-4 \sum_{i<j}\left(K_{i j}+K_{i j^{*}}\right) \zeta_{i i^{*}} \zeta_{j j^{*}} \\
& =\sum_{i<j}\left(K_{i j}+K_{i j^{*}}\right)\left(\zeta_{i i^{*}}-\zeta_{j j^{*}}\right)^{2} .
\end{aligned}
$$

For, in terms of a $\phi$-basis $\left\{X_{\alpha}\right\}, \alpha=0,1, \cdots, n, 1^{*}, \cdots, n^{*}, R_{\alpha 0 \beta 0}=0$, so

$$
\begin{aligned}
\sum_{\alpha, \beta, \gamma} R_{\alpha \beta} \zeta_{\alpha \gamma} \zeta_{\beta \gamma}= & -\sum_{\alpha, \beta, \gamma, \sigma} R_{\alpha \sigma \beta \sigma} \zeta_{\alpha \gamma} \zeta_{\beta \gamma} \\
& =2 \sum_{\substack{i \\
j \neq i, i^{*}}}\left(K_{i j}+K_{i j^{*}}\right) \zeta_{i i^{*}} \zeta_{j j^{*}} .
\end{aligned}
$$

Setting $\zeta=\Phi$ in formula (4.1), it is seen that $F(\zeta)=0$. Consequently, $\nabla \Phi$ vanishes, so $M$ is normal, that is, it is cosympletic.

The proof of Corollary 1.1 is an immediate consequence of the fact that $R(X, Y)$ vanishes.

Let $\widetilde{S}$ be the 2-form defined by

$$
\widetilde{S}(X, Y)=S(X, \phi Y),
$$

and let $\psi$ be the 2 -form given by $(1 / 2) \iota(\Phi) R$. Then, we have

CoROLlaRY 1.2. Let $M$ be an almost cosymplectic manifold. If

$$
\psi=\widetilde{S},
$$

then $M$ is cosymplectic.

COROLlaRY 1.3. The same conclusion prevails if

$$
\iota(\Phi)(\widetilde{S}-\psi)=0,
$$

that is, if

$$
r=S(\xi, \xi)+\iota(\Phi) \psi
$$


where $r$ is the scalar curvature.

Proposition 3. If the fundamental vector field of a 3-dimensional almost cosymplectic manifold $M(\phi, \eta, g)$ is a Killing field, then $M$ is cosymplectic.

Proof. We must show that $M$ is normal. To this end, observe that $L_{\xi} \Phi=d \iota(\xi) \Phi+\iota(\xi) d \Phi=0$, where $L_{X}$ is the Lie derivative operator. For, $\Phi$ is closed and $\iota(\xi) \Phi=0$. On the other hand, since $L_{\xi} g=0, L_{\xi} \phi$ vanishes.

Let $X, \phi X, \xi$ be an orthonormal set of vector fields. Then, we have three cases to examine in formula (2.4).

Case (a). $Y=\phi X$. Then, since $\eta(X)=0$

$$
\begin{aligned}
{[\phi, \phi](X, \phi X)=} & {\left[\phi X, \phi^{2} X\right]-\phi[\phi X, \phi X]-\phi\left[X, \phi^{2} X\right]+\phi^{2}[X, \phi X] } \\
= & {[\phi X,-X+\eta(X) \xi]-\phi[X,-X+\eta(X) \xi] } \\
& -[X, \phi X]+\eta([X, \phi X]) \xi \\
= & \eta([X, \phi X]) \xi \\
= & 0,
\end{aligned}
$$

the latter following since $\eta$ is closed and $\eta \circ \phi=0$.

Case (b). $Y=\xi$. Then,

$$
\begin{aligned}
{[\phi, \phi](X, \xi) } & =[\phi X, \phi \xi]-\phi[\phi X, \xi]-\phi[X, \phi \xi]+\phi^{2}[X, \xi] \\
& =\phi([\xi, \phi X]-\phi[\xi, X]) \\
& =\phi\left(L_{\xi} \phi\right) X \\
& =0 .
\end{aligned}
$$

Case ( c).

$$
\begin{aligned}
{[\phi, \phi](\phi X, \xi) } & =\left[\phi^{2} X, \phi \xi\right]-\phi\left[\phi^{2} X, \xi\right]-\phi[\phi X, \phi \xi]+\phi^{2}[\phi X, \xi] \\
& =-\phi[-X+\eta(X) \xi, \xi]+\phi^{2}[\phi X, \xi] \\
& =\phi[X, \xi]-[\phi X, \xi]+\eta([\phi X, \xi]) \xi \\
& =-\left(L_{\xi} \phi\right) X-\eta\left(\left(L_{\xi} \phi\right) X+\phi L_{\xi} X\right) \\
& =0 .
\end{aligned}
$$

Observe that in a coordinate neighborhood with the coordinate vectors $X, Y, Z, W$, if $R(X, Y) \dot{\phi}=\phi R(X, Y)$, then

$$
\begin{aligned}
-\left(\left[\nabla_{X}, \nabla_{Y}\right] \Phi\right)(Z, W) & =\Phi\left(\left[\nabla_{X}, \nabla_{Y}\right] Z, W\right)+\Phi\left(Z,\left[\nabla_{X}, \nabla_{Y}\right] W\right) \\
& =g(R(X, Y) \phi Z, W)+g(R(X, Y) Z, \phi W),
\end{aligned}
$$


by (3.1), so an equivalent formulation of the integrability condition of Theorem 1 is given by

$$
R(X, Y) \Phi=0
$$

where the curvature transformation acts on the 2 -form $\Phi$ as a derivation.

To construct an almost cosymplectic structure on $M \times R$ or $M \times S^{1}$ where $M$ is an almost Kaehler manifold take any point $(m, t)$ of either space and set $\phi(X, Y)=(J X, 0), X \in M_{m}, Y \in R_{t}$ or $S_{t}^{1}, \xi=(0, d / d t)$ and $\eta=(0, d t)$ where $J$ is the almost complex structure of $M$.

5. Integrability of almost Sasakian structures. Let $M(\phi, \eta, g)$ be a normal contact metric space with structure tensors $\phi, \eta$ and $g$. Since $M$ is normal, $\xi$ is a Killing field with respect to $g$, so by definition $M$ is an almost Sasakian manifold. The fact that $\xi$ is a Killing field also yields the well-known second order condition

$$
\Delta \eta=2 \iota(\eta) S \text {. }
$$

Again, by the normality of $M$, the contact form also satisfies the second order differential equation

$$
\left(\nabla_{Z} \nabla_{X} \eta\right)(Y)=\left(\nabla_{Z} \Phi\right)(X, Y)=g(Y, Z) \eta(X)-g(X, Z) \eta(Y) .
$$

Substituting (5.2) into (5.1), we find

$$
\iota(\eta) S=-n \eta
$$

Forming the codifferential of $\Phi$, we also obtain from (5.2)

$$
\delta \Phi=2 n \eta \text {. }
$$

Observe that $\eta$ is coclosed, whereas in a cosymplectic manifold, it is closed. If $M$ is compact,

$$
V=\frac{1}{4 n^{2}} \int_{M} \eta \wedge * \eta
$$

where $V$ is the volume of $M$.

We denote by $Q \Phi$ the 2 -form with values

$$
Q \Phi(X, Y)=\frac{1}{2}[\widetilde{S}(Y, X)-\widetilde{S}(X, Y)]
$$

Expressing $d \Phi$ in terms of covariant derivatives, then applying $\nabla$, employing the Ricci interchange formula, and finally using the first Bianchi identity, the following decomposition of the fundamental 2 -form is obtained 


$$
4 n \Phi=\delta \nabla \Phi+2 Q \Phi+2 \psi
$$

by virtue of (2.3) and (5.3). Substituting (5.2) into (5.4), we find

$$
Q \Phi=(2 n-1) \Phi-\psi \text {. }
$$

Conversely, suppose that $M(\phi, \eta, g)$ is an almost Sasakian space and (5.5) holds. Then, by equation (5.4)

$$
\delta \nabla \Phi=2 \Phi
$$

But, since $|\Phi|^{2}=2 n$

$$
\frac{1}{2} \Delta|\Phi|^{2}=\langle\delta \nabla \Phi, \Phi\rangle-|\nabla \Phi|^{2}=0,
$$

so that

$$
|\nabla \Phi|^{2}=2|\Phi|^{2}=4 n
$$

On the other hand, by setting

$$
\Pi(X, Y, Z)=\left(\nabla_{X} \Phi\right)(Y, Z)+g(X, Y) \eta(Z)-g(X, Z) \eta(Y)
$$

and computing its square length,

$$
|I I|^{2}=|\nabla \Phi|^{2}-4 \iota(\xi) \delta \Phi+4 n=0,
$$

from which $\Pi=0$, so $M$ is normal, that is Sasakian. Thus, we have proved

THEOREM 4. In order that an almost Sasakian manifold be Sasakian, it is necessary and sufficient that

$$
Q \Phi=(2 n-1) \Phi-\psi \text {. }
$$

CoROLLARY 4.1. If the metric $g$ of an almost Sasakian manifold $M(\phi, \eta, g)$ is an Einstein metric, that is, if $S=\lambda g$ (or, if $S=$ $\lambda(g-\eta \otimes \eta)$, and if $\psi=(2 n-\lambda-1) \Phi$, then $M$ is Sasakian.

\section{BIBLIOGRAPHY}

1. D. E. Blair, The theory of Quasi-Sasakian structures, J. Differential Geometry 1 (1967), 331-345.

2. D. E. Blair and S. I. Goldberg, Topology of almost contact manifolds, J. Differential Geometry 1 (1967), 347-354.

3. S. I. Goldberg, Integrability of almost Kaehler manifolds, Proc. Amer. Math. Soc. 21 (1969), 96-100.

4. A. Gray, Vector cross products on manifolds, Trans. Amer. Math. Soc. (to appear) 
5. E. M. Moskal, Contact manifolds of positive curvature, Thesis, University of Illinois, (1966).

Received December 9,1968. This research was partially supported by the National Science Foundation. The second author is a G. A. Miller Visiting Professor at the University of Illinois.

UNIVERSITY OF ILLINOIS

URBANA, ILLINOIS 


\section{PACIFIC JOURNAL OF MATHEMATICS}

\section{EDITORS}

\author{
H. ROYDEN \\ Stanford University \\ Stanford, California \\ Richard Pierce \\ University of Washington \\ Seattle, Washington 98105
}

\author{
J. DugundJI \\ Department of Mathematics \\ University of Southern California \\ Los Angeles, California 90007 \\ BASIL GoRDON \\ University of California \\ Los Angeles, California 90024
}

\section{ASSOCIATE EDITORS}
E. F. BECKENBACH
B. H. Neumann
F. WOLF
K. YoSHIDA

\section{SUPPORTING INSTITUTIONS}

\author{
UNIVERSITY OF BRITISH COLUMBIA \\ CALIFORNIA INSTITUTE OF TECHNOLOGY \\ UNIVERSITY OF CALIFORNIA \\ MONTANA STATE UNIVERSITY \\ UNIVERSITY OF NEVADA \\ NEW MEXICO STATE UNIVERSITY \\ OREGON STATE UNIVERSITY \\ UNIVERSITY OF OREGON \\ OSAKA UNIVERSITY \\ UNIVERSITY OF SOUTHERN CALIFORNIA
}

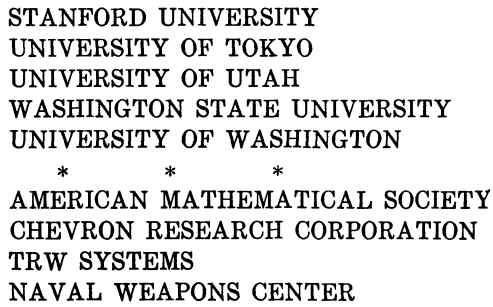

The Supporting Institutions listed above contribute to the cost of publication of this Journal, but they are not owners or publishers and have no responsibility for its content or policies.

Mathematical papers intended for publication in the Pacific Journal of Mathematics should be in typed form or offset-reproduced, double spaced with large margins. Underline Greek letters in red, German in green, and script in blue. The first paragraph or two must be capable of being used separately as a synopsis of the entire paper. It should not contain references to the bibliography. Manuscripts, in duplicate if possible, may be sent to any one of the four editors. Please classify according to the scheme of Math. Rev. 36, 1539-1546. All other communications to the editors should be addressed to the managing editor, Richard Arens, University of California, Los Angeles, California, 90024.

50 reprints are provided free for each article; additional copies may be obtained at cost in multiples of 50 .

The Pacific Journal of Mathematics is published monthly. Effective with Volume 16 the price per volume (3 numbers) is $\$ 8.00$; single issues, $\$ 3.00$. Special price for current issues to individual faculty members of supporting institutions and to individual members of the American Mathematical Society: $\$ 4.00$ per volume; single issues $\$ 1.50$. Back numbers are available.

Subscriptions, orders for back numbers, and changes of address should be sent to Pacific Journal of Mathematics, 103 Highland Boulevard, Berkeley, California, 94708.

PUBLISHED BY PACIFIC JOURNAL OF MATHEMATICS, A NON-PROFIT CORPORATION

Printed at Kokusai Bunken Insatsusha (International Academic Printing Co., Ltd.), 7-17, Fujimi 2-chome, Chiyoda-ku, Tokyo, Japan. 


\section{Pacific Journal of Mathematics}

\section{Vol. 31, No. $2 \quad$ December, 1969}

Efraim Pacillas Armendariz, Quasi-injective modules and stable torsion

classes..........................................

J. Adrian (John) Bondy, On Ulam's conjecture for separable graphs...

Vasily Cateforis and Francis Louis Sandomierski, On commutative rings over which the singular submodule is a direct summand for every module .....

Rafael Van Severen Chacon, Approximation of transformations with continuous

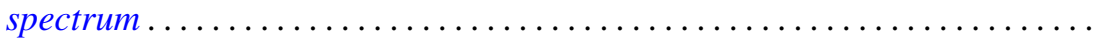

Raymond Frank Dickman and Alan Zame, Functionally compact spaces ...... 303

Ronald George Douglas and Walter Rudin, Approximation by inner

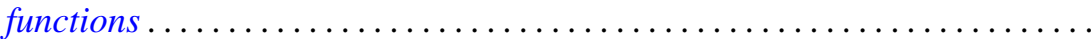

John Walter Duke, A note on the similarity of matrix and its conjugate

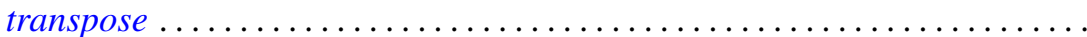

Micheal Neal Dyer and Allan John Sieradski, Coverings of mapping

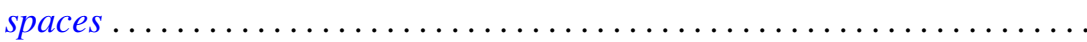

Donald Campbell Dykes, Weakly hypercentral subgroups of finite groups .....

Nancy Dykes, Mappings and realcompact spaces.....................

Edmund H. Feller and Richard Laham Gantos, Completely injective

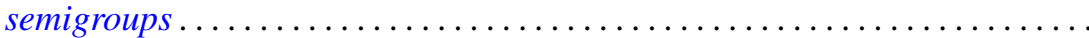

Irving Leonard Glicksberg, Semi-square-summable Fourier-Stieltjes

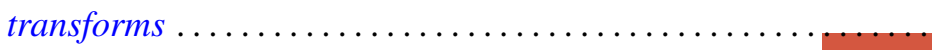

Samuel Irving Goldberg and Kentaro Yano, Integrability of almost cosymplectic structures...

Seymour Haber and Charles Freeman Osgood, On the sum $\sum\langle n \alpha\rangle^{-t}$ and numerical integration ..........................

Sav Roman Harasymiv, Dilations of rapidly decreasing functions ....

William Leonard Harkness and R. Shantaram, Convergence of a sequence of

transformations of distribution functions

Herbert Frederick Kreimer, Jr., A note on the outer Galois theory of rings ...

James Donald Kuelbs, Abstract Wiener spaces and applications to analysis. .

Roland Edwin Larson, Minimal $T_{0}$-spaces and minimal $T_{D}$-spaces...

A. Meir and Ambikeshwar Sharma, On Ilyeff's conjecture .

Isaac Namioka and Robert Ralph Phelps, Tensor products of compact convex sets....

James L. Rovnyak, On the theory of unbounded Toeplitz operators ....

Benjamin L. Schwartz, Infinite self-interchange graphs.......

George Szeto, On the Brauer splitting theorem...

Takayuki Tamura, Semigroups satisfying identity $x y=f(x$,

Kenneth Tolo, Factorizable semigroups .................. 